\title{
Nonpersistent endocrine disrupting chemicals and reproductive health of women
}

\author{
Yeon Jean Cho, MD, PhD', Jeong Hye Yun, MD', Su Jin Kim, MD', Hyun Young Kwon, $M D^{2}$ \\ ${ }^{1}$ Department of Obstetrics and Gynecology, Dong-A University Medical Center, Dong-A University, College of Medicine, Busan; ${ }^{2}$ Mirae Womens \\ Hospital, Jinju, Korea
}

Nonpersistent endocrine disrupting chemicals (npEDCs) are exogenous chemicals or mixtures of industrial agents that can interfere with the normal action of hormone with a shorter half-life and lower liposolubility. These are commonly found in plastics, medical equipment, detergents, and cosmetics. Recently, role of npEDCs on the changes of ovary and/or uterus development and alterations in hormonal signaling has been emphasized. However, many controversial results exist on the effects of npEDCs and reproductive health of women. Thus, we have focused to review the scientific evidence of a causal relationship between exposure to npEDCs and representative female reproductive issues such as menstrual cycle, endometriosis, uterine fibroids, polycystic ovarian syndrome and infertility/subfertility. Though not all studies indicated a positive correlation of npEDCs with female reproductive issues, the reviewed data illustrated that the majority of the available data strengthen the evidence of reproductive health-related actions of npEDCs. In future, recommendations should be made in order to reduce human exposure to npEDCs and to protect from steadily increasing reproductive health risks.

Keywords: Endocrine disruptors; Endometriosis; Uterine fibroid; Polycystic ovarian syndrome; Infertility

\section{Introduction}

Endocrine disrupting chemicals (EDCs) are chemicals that interfere with the body's endocrine system and cause adverse developmental, reproductive, neurological, and immune effects. EDCs may mimic, in whole or in part, natural body hormones that exhibit their effects by acting on specific receptor proteins. They also bind to cell receptors, thereby blocking their interaction with natural hormones, or alter hormone metabolism. Individual EDCs may interact with more than one receptor, and multiple EDCs can interact with the same receptor, highlighting the unusual properties of environmental EDCs [1]. We can divide these chemicals according to their lipophilic nature. Chemicals with high lipophilic activity and a longer half-life are called 'persistent EDC' or 'persistent organic pollutants' (POPs), which may bioaccumulate in fat and can be biomagnified through the food chain. Representative chemicals are dioxins, dichlorodiphenyl-trichloroethylene, heptachlor, and polychlorinated biphenyls. For POPs, many epidemiologic and in vitro studies provided estimates of the gynecologic health risks in human populations [2-8].

There are other chemicals with a shorter half-life and lower liposolubility, which are called nonpersistent EDCs (npEDCs). Examples of npEDCs are bisphenol A (BPA), phthalates, parabens, and triclosans (TCSS). BPA (4,4'-(propane-2,2-diyl) diphenol) is a well-known EDC component of baby bottles, children's toys, dental sealants, coating of receipts, and epoxy resins used to coat the inside of food cans. Di-2-ethylhexyl phthalate (DEHP) is used as a plasticizer and is contained in a wide range of products such as plastics, cosmetics, and medical devices. Parabens (alkyl esters of 4-hydroxy benzoic

Received: 2019.02.28. Revised: 2019.07.14. Accepted: 2019.07.24. Corresponding author: Yeon Jean Cho, MD, PhD

Department of Obstetrics and Gynecology, Dong-A University Medical Center, Dong-A University College of Medicine,

26 Daesingongwon-ro, Seo-gu, Busan 49201, Korea

E-mail: jeanjane@dau.ac.kr

https://orcid.org/0000-0003-2755-4970

Articles published in Obstet Gynecol Sci are open-access, distributed under the terms of the Creative Commons Attribution Non-Commercial License (http://creativecommons. org/licenses/by-nc/3.0/) which permits unrestricted non-commercial use, distribution, and reproduction in any medium, provided the original work is properly cited.

Copyright $\odot 2020$ Korean Society of Obstetrics and Gynecology 


\section{Obstetrics \& Gynecology Science}

Vol. 63, No. 1, 2020

acid) are used as preservatives in personal care products such as cosmetics and pharmaceutical products. TCS is an antimicrobial compound used in consumer products such as toothpaste, mouth wash, and hand sanitizers. In contrast to POPs, the exact effect of npEDCs on gynecologic health risk is not totally understood. These chemicals may cause subtle changes in ovary and/or uterus development and alterations in hormonal signaling, possibly resulting in variable phenotypes [9]. Although the direct effect of npEDCs on these conditions is difficult to prove, low-dose and continuous environmental exposure might play a critical role on gynecologic health. The purpose of this article was to review the scientific evidence of a causal relationship between exposure to npEDCs and representative female reproductive issues, menstrual cycle, endometriosis, uterine fibroid (UF), polycystic ovarian syndrome (PCOS), and infertility/subfertility.

\section{Menstrual cycle}

\section{Bisphenol A}

In rodents, the effects of BPA exposure on estrous cycle were examined in several studies [10-13]. Uterine exposure to BPA alters several apoptotic factors and causes germ cell nest breakdown, which results in estrous cycle changes [10]. BPA treatment significantly decreases serum estradiol (E2) concentration, which is accompanied by increased duration of the estrus phase, increased ovarian cell apoptosis, and decreased E2-regulated protein expression and collagen content in the uterus [11]. In contrast, high-dose BPA shortens the estrous cycle day and length [12]. Neonatal exposure to BPA leads to abnormal function of the neural network that controls the cycle. Hypothalamic LH-releasing hormone premRNA processing and steroid receptor expression in nuclei controlling estrous cyclicity are permanently disrupted [13].

In humans, urinary BPA metabolites were measured in 221 healthy women and BPA was associated with shorter luteal phase. However, no association was seen in follicular phase length [14]. Analysis of the longitudinal urine samples from healthy, premenopausal women showed that BPA was associated with increased E2, which may influence the menstrual cycle [15]. Only a few associations have been reported between BPA exposure and changes in menstrual characteristics. The effects of BPA exposure on estrous cyclicity in rodents and menstrual cycle in humans are still inconclusive.

\section{Phthalates}

In rodents, DEHP exposure decreased the levels of estrogen and progesterone, in addition to prolonging menstrual cycles and anovulation [16]. High-dose DEHP treatments resulted in reduced serum estradiol, prolonged estrous cycles, and inhibition of ovulation [17]. Continuous exposure to DEHP during adult life prolonged the duration of estrous cycle. Low levels of DEHP disrupted phosphatidylinositol 3-kinase signaling, which resulted in abnormal estrous cycles and primordial follicle recruitment [18]. DEHP treatment showed decreased estradiol production in cultured rat ovarian tissues [19]. In cultured mouse antral follicle DEHP and mono-(2-ethylhexyl)phthalate (MEHP) inhibited estradiol biosynthesis and inhibited mRNA expression of cyclin-D-2, cyclin-dependentkinase-4, and aromatase (Arom) [20]. MEHP also acts on the granulosa cells by decreasing the level of cyclic adenosine monophosphate stimulated by follicle stimulating hormone and by activating peroxisome proliferator-activated receptors (PPARs), which leads to decreased Arom transcription [21]. These may suggest that phthalates alter E2 production through the decreased expression of cell cycle regulators and specific receptor-mediated responses.

In humans, high concentrations of urinary monocarboxyoctyl phthalate are associated with short luteal phase. But menstrual cycle-specific estimates of urinary phthalate metabolites were not associated with the follicular-phase length [14]. Another study did not show a consistent relationship between menstrual phase and phthalate metabolite concentrations [22]. Based on described studies, a mechanistic model explaining phthalate effects on menstrual cycle has been proposed. However, the exact effects of phthalates on menstrual cycle are inconclusive.

\section{Parabens and triclosan}

Parabens and paraben metabolites are associated with increased E2 levels in healthy premenopausal women. In Japanese women, butyl paraben concentrations (odds ratio [OR], 0.83; $95 \%$ confidence interval [Cl], 0.70-0.99) are negatively correlated with menstrual cycle length and urinary estrogenequivalent total paraben concentrations $(\mathrm{OR}, 0.73 ; 95 \% \mathrm{Cl}$, 0.56-0.96) [23]. To date, no studies have addressed the effects of TCS on the menstrual cycle. 


\section{Obstetrics \& Gynecology Science}

Yeon Jean Cho, et al. npEDCs affect reproductive health

\section{Endometriosis}

Endometriosis has a multifactorial etiology involving genetic, hormonal, immunologic, and environmental factors [24]. As for other reproductive disorders, a direct causality between $n p E D C s$ and endometriosis is difficult to prove. Moreover, the experimental and epidemiological data are not always consistent. EDCs are of particular interest as potential contributors to endometriosis because they can alter steroidogenesis, immunologic function, and are epigenetic causal factors involved in this disease progression [25].

\section{Bisphenol A}

As rodents do not develop spontaneous endometriosis, experimental models are used to study the relationship between BPA and endometriosis. Oral administration of BPA increases gland nest density and periglandular collagen accumulation, characteristics of an endometriosis-like phenotype, in adult CD-1 mice. These have shown increased collagen I and III expression and decreased matrix metalloproteinase (MMP)-2 and MMP-14 expression in those tissues around the uterus $[26,27]$. Prenatal exposure of mouse to BPA induces an endometriosis-like phenotype in female offspring. Moreover, the effects of EDCs during critical developmental stages seem to be long-lasting [28]. In these mice, primordial and developing follicle numbers were significantly lower than those in controls [29]. These results indicate that EDCs may induce endometriotic phenotype and compromise ovarian function of the following generations.

In humans, the serum of patients with endometriosis is known to contain at least 1 of the 2 bisphenols (BPA and bisphenol B) [30]. One population-based case-control study revealed the median creatinine-corrected total urinary BPA concentrations were higher $(1.32 \mu \mathrm{g} / \mathrm{g}$, interquartile range [IQR], 0.79-2.21) in endometriosis patients than in controls (1.24 $\mu \mathrm{g} / \mathrm{g}, \mathrm{IQR}, 0.65-2.54)$. Especially for non-ovarian pelvic endometriosis, statistically significant positive associations were observed with urinary BPA concentrations [31]. In patients with ovarian endometrioma, the mean urinary concentration of BPA was found to be significantly higher than that in control subjects $(5.53 \pm 3.47 \mathrm{ng} / \mathrm{mL}$ vs. $1.43 \pm 1.57 \mathrm{ng} / \mathrm{mL})$ [32]. Some other epidemiologic studies have shown a positive association between urinary BPA levels and endometriosis $[8,30,33]$. In contrast, a cross-sectional study in Japanese and USA patients found no significant associations between endometriosis and urinary BPA concentrations [34,35]. Thus, not all studies have shown positive associations.

Low BPA concentrations affect human endometrial stromal cell (ESC) physiology. BPA enhanced progesterone-induced decidualization and promoted ESC migration and oxidative stress in vitro $[36,37]$. Currently, the molecular mechanisms involved in the progression of endometriosis are not well understood. Moreover, studies using parenteral routes of administration may have limited relevance, if any, to human risk assessment. Thus, the impact of BPA on reproduction is still unclear and epidemiologic data in humans are limited.

\section{Phthalates}

Earlier studies have analyzed the levels of phthalates in serum in relation to the risk of endometriosis. Cobellis et al. [38] were the first to demonstrate significantly higher plasma concentrations of DEHP in endometriotic women than in controls. In Korea, women with advanced endometriosis exhibited significantly higher plasma levels of MEHP and DEHP than those in control women without endometriosis [39]. Similar results were observed in Indian women $[40,41]$.

The urinary concentration of phthalate metabolites is commonly used as a representative biomarker of exposure to DEHP because, after oral administration of DEHP, about $75 \%$ of the compound is excreted in urine in the form of DEHP metabolites after 44 hours in humans [42]. In the National Health and Nutrition Examination Survey (NHANES), a crosssectional study of urinary phthalates revealed a significantly positive association between mono-n-butyl phthalate (MBP) and the risk of endometriosis [43]. Moreover, specific phthalates were associated with MRI-diagnosed endometriosis [34]. In the Endometriosis, Natural History, Diagnosis, and Outcomes Study, 6 phthalate metabolites [MBP, mono-[(2carboxymethyl) hexyl] phthalate, mono (2-ethyl-5-carboxyphentyl) phthalate, MEHP, mono (2-ethyl-5-hydroxyhexyl) phthalate (MEHHP), and mono (2-ethyl-5-oxohexyl) phthalate) (MEOHP) were significantly associated with increased odds of endometriosis [34].

In contrast to the above studies, a population-based casecontrol study conducted in the USA as part of the Women's Risk of Endometriosis study failed to demonstrate the association between specific phthalate metabolites and endometriosis risk [44], while a study in Japanese women failed to demonstrate a positive association between urinary concentrations of phthalate metabolites and the risk of endometriosis [45]. 


\section{Obstetrics \& Gynecology Science}

Vol. 63, No. 1, 2020

Some in vitro studies suggested that exposure to phthalates might play a role in the establishment of endometriosis. In particular, DEHP promotes the viability of ESCs [46], and treatment of endometrial cells with DEHP leads to significant increases in MMP-2 and MMP-9 activities, cellular invasiveness, extracellular signal-regulated kinase (ERK) phosphorylation, and p21-activated kinase 4 expression [47]. In human ESCs, DEHP exposure increases p-ERK/p-p38- and nuclear factor-kB-mediated transcription through an oxidative stress pathway. Moreover, DEHP induces the expression of estrogen receptor-a (ERa) in a dose-dependent manner [48]. Notably, in the endometrium of patients with endometriosis, DEHP induces aldo-keto reductase activity, which is involved in prostaglandin synthesis and progesterone-resistance [49]. Although in vitro results support the hypothesis that phthalates may be an inducer of endometriosis, further investigations will be required to definitively establish the association between phthalates and endometriosis.

\section{Parabens and triclosan}

ESCs exposed to TCS showed increased decidualization effect which suggested than TCS may alter the nature of normal ESCs [36]. That is the only study about TCS effects in relation to endometriosis, and no studies have addressed the possible relationship between paraben and endometriosis.

\section{Uterine fibroid}

UF is one of the most frequent gynecologic tumors among women of reproductive age and causes symptoms such as abnormal uterine bleeding, menorrhagia, and pelvic pain. It is known as an estrogen-dependent disease, and a possible involvement of EDCs has been suggested [50]. We here describe the effect of BPA and phthalate on UF. There are no studies about paraben and TCS with UF.

\section{Bisphenol A}

In China and the USA, the mean urine BPA concentration was significantly higher in the UF group than in controls $[15,51,52]$. In contrast, Korean studies revealed that the serum concentration of BPA is not related to UF progression $[53,54]$. Thus, epidemiological studies on BPA and UF are not consistent.

An in vitro study showed that BPA promoted the growth of UF cells and the expression of ERa, insulin-like growth factor-1, and vascular endothelial growth factor in a dose and time-dependent manner [55]. BPA seems to promote the proliferation of UF cells via the ERa and transforming growth factor- $\beta$ signaling pathways [56]. At environmentally relevant doses, BPA enhanced cell proliferation, induced cyclooxygenase-2 (COX-2) gene expression, and promoted cell migration and invasiveness [57]. BPA induced proliferation in immortalized human UF cells through membrane-associated ERa36 by the activation of Src, epidermal growth factor receptor, Ras, and microtubule affinity regulating kinase pathways [58]. Most of these in vitro studies indicated that BPA increased the proliferation of human UF cells, possibly contributing to UF growth.

\section{Phthalates}

The NHANES from 1999-2004 showed a positive association between a specific phthalate metabolite (MBP) and the risk of UFs. However, other metabolites (MEHP, MEHHP, and MEOHP) were inversely associated with UFs [43]. Women with UFs exhibited significantly higher levels of total urinary MEHP than those in healthy controls [59]. In one populationbased study conducted in Korea, the urinary concentration of 16 phthalate metabolites was compared in women with and without UF. Using multiple logistic regression analyses, a significant association was found between the levels of total urinary DEHP metabolites and UF [60]. Recently, in the USA, a cross-sectional study on premenopausal women seeking surgical care for UFs showed elevated urine concentrations of several phthalates, which were positively associated with the uterine volume. In particular, these effects were more prominent for DEHP metabolites [61].

In vitro treatment of DEHP promoted cell viability, proliferative activity, and anti-apoptotic activity in human leiomyoma cells. Furthermore, DEHP treatment on human UF cells increased hypoxia inducible factor $1 \mathrm{a}$ and COX-2 expression, which may be involved in inflammatory response [62]. These findings suggest an association between phthalate exposure and UF. Despite the importance of these reports, additional phthalates should be tested for association with UFs.

\section{Polycystic ovarian syndrome}

PCOS is one of the most common ovulatory disorders with 


\section{Obstetrics \& Gynecology Science}

Yeon Jean Cho, et al. npEDCs affect reproductive health

hyperandrogenemia and/or insulin resistance. Recently, there is emerging evidence about the effect of npEDCs on PCOS, especially BPA. However, data regarding the impact of phthalate, TCS, and paraben exposure on PCOS are very limited.

\section{Bisphenol A}

After a positive relationship between androgen concentration and BPA in women with ovulatory dysfunction has been suggested [63], a case control study (71 women with PCOS and 100 women without PCOS) found that serum BPA was significantly higher in PCOS women and that there was a significant positive association between BPA, androgen concentration, and insulin resistance [64]. Furthermore, PCOS women with higher serum BPA had more severe insulin resistance, increased free androgen index, and increased markers of chronic inflammation [65]. Market seller women with PCOS exhibited higher serum BPA levels than non-PCOS women [66]. According to a recent meta-analysis, PCOS patients had significantly higher BPA levels than those of control groups (standardized mean difference, 2.437; 95\% Cl, 1.265-3.609, $P<0.001)$ [67]. These results suggest a role of BPA in the development and/or pathogenesis of PCOS.

BPA may act on ERs to mimic actions like estrogen and may bind to membrane receptors to cause harmful effects even at pico- and nanomolar concentrations. Rutkowska and Diamanti-Kandarakis [68] summarized the molecular effect of BPA on PCOS as altering ovarian steroidogenesis, aggravating hyperandrogenism state, altering oocyte development and folliculogenesis, and worsening metabolic parameters such as insulin resistance, obesity, oxidative stress, and inflammation.

\section{Phthalates}

PCOS patients have significantly lower urinary concentrations of monobenzyl phthalate (mBzP), and low urine concentrations of $\mathrm{MBzP}$ and MBP increase the likelihood of PCOS (OR, 0.14-0.25; $P<0.05)$. This result only showed that PCOS patients may differ from controls in their environmental contaminant profile but failed to show a positive correlation between phthalate and PCOS [69]. In order to verify the impact of antenatal exposure to phthalates on the development of PCOS in the descendants, a study in the context of the Western Australian Pregnancy Cohort (Raine) Study assessed the most common phthalate metabolites in 3,000 pregnant women. This study showed antenatal exposure to phthalates had some protective effects on the development of PCOS which implicated long-term effects of phthalates on reproduction [70]. In contrast to these results, one study suggested that gestational exposure to some phthalates (dibutyl phthalate and DEHP) results in polycystic ovaries and hormonal profiles similar to PCOS [71]. To summarize, only limited and contradictory results are available regarding the effect of phthalates on PCOS.

\section{Parabens and triclosan}

In a cross-sectional study in Chinese infertile women, patients with PCOS exhibited significantly higher TCS concentrations than those in control women (median of TCS (IQR), $\mu \mathrm{g} / \mathrm{g}$ creatinine: 1.49 (0.68-3.80) vs. 1.06 (0.52-3.02), $P=0.0407)$. Compared to the lowest tertile, the highest tertile of TCS concentration was associated with an increased odds of PCOS (OR, 1.99; 95\% Cl, 1.05-3.79) [72]. In contrast, a casecontrol study exploring the association between the urinary concentration of personal care products and PCOS found no significant differences in TCS detection rate or the total concentration of analytes [73].

\section{Infertility and/or subfertility}

Female infertility is a complex disorder that can be caused by anatomic, genetic, environmental, and endocrine factors. The comprehension of npECD mechanisms of action, as well as the presumed risks deriving from the exposure to these compounds, may be crucial to improve women's fertility.

\section{Bisphenol A}

Numerous studies have investigated the effect of BPA on women infertility. BPA is associated with women infertility by affecting the morphology and functions of the oviduct, uterus, and ovary [74]. Furthermore, BPA affects the hypothalamus-pituitary-ovarian function by altering the secretion of gonadotropin-releasing hormones in the hypothalamus and promoting pituitary proliferation [75]. The Shanghai Birth Cohort Study investigated the impact of BPA exposure on fecundability in healthy women. Each one-unit increase in urinary concentrations of BPA was associated with a $13 \%$ reduction in fecundability (fecundability $\mathrm{OR}, 0.87 ; 95 \% \mathrm{Cl}$, $0.78-0.98$ ) and a $23 \%$ increase in the odds of infertility 


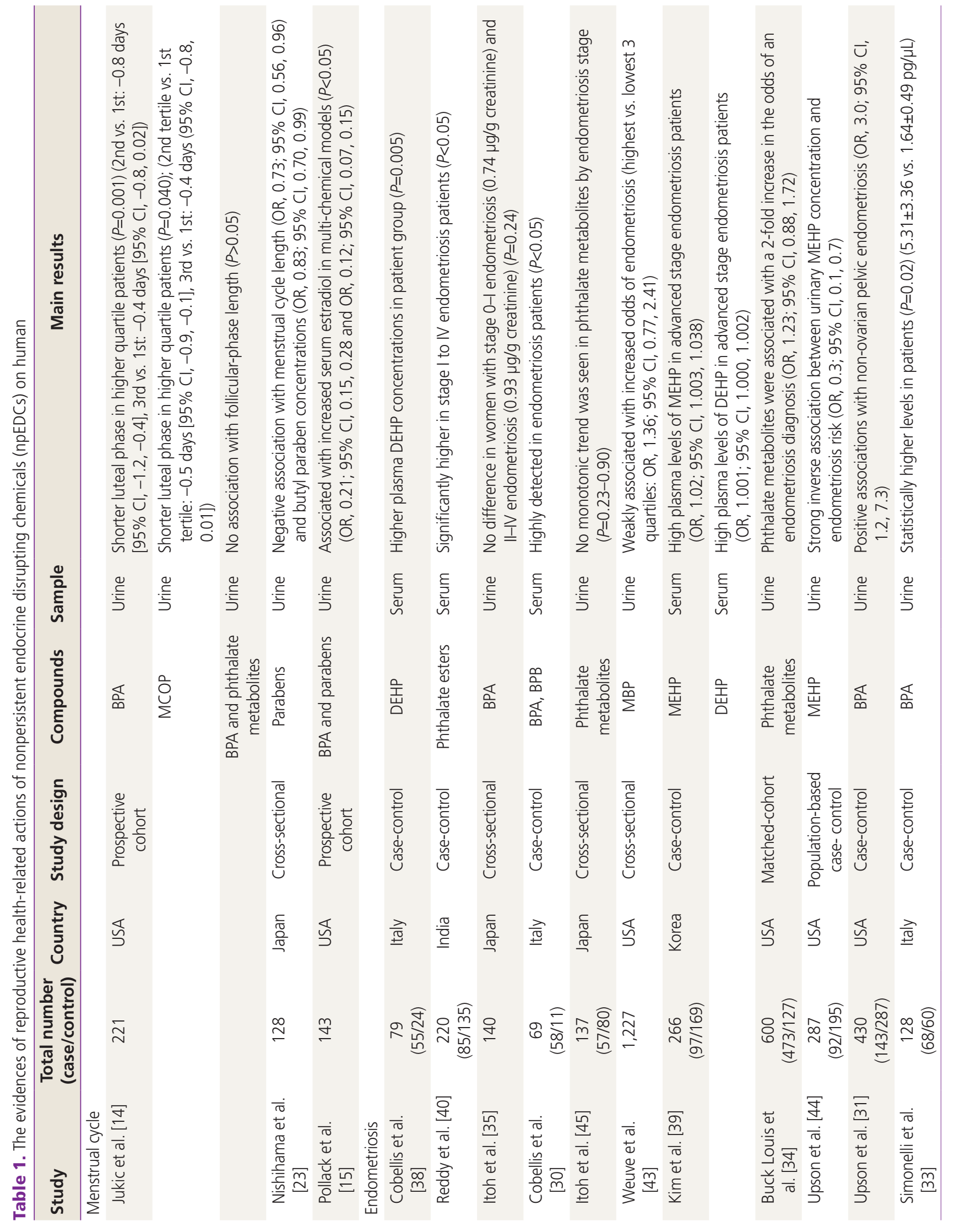




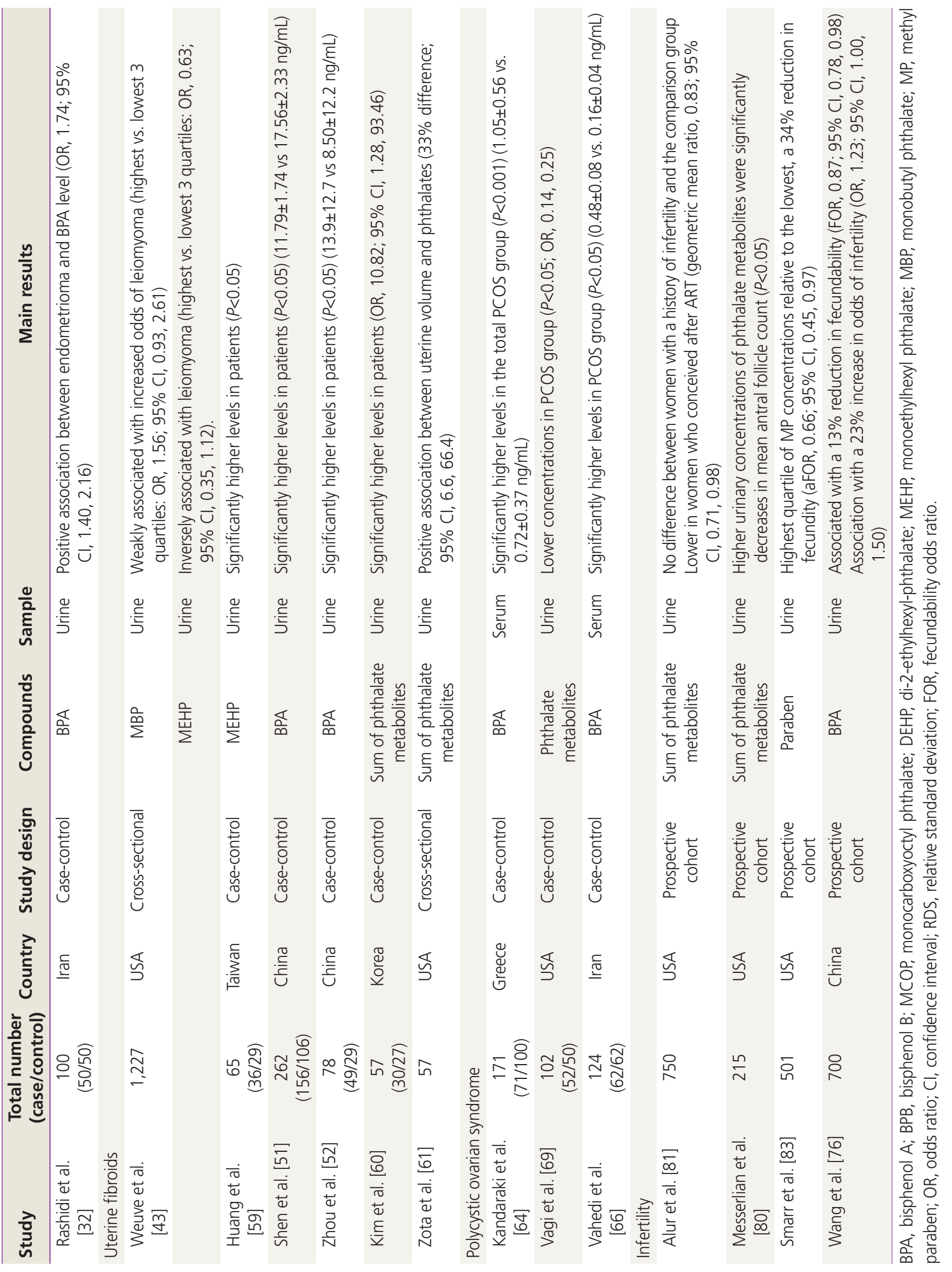




\section{Obstetrics \& Gynecology Science}

Vol. 63, No. 1, 2020

(OR, 1.23; $95 \% \mathrm{Cl}, 1.00-1.50)$. In addition, these associations were strengthened among women over 30 years of age [76].

Recently, because of the potential harmful effects of BPA, many countries replaced BPA with the analogs bisphenol $S$ (BPS) and bisphenol $F$ (BPF). The products containing these analogs are known as 'BPA-free products'. However, several in vitro and in vivo studies demonstrated that BPS and BPF also exert the same endocrine disrupting effects as BPA [77]. Regarding oocyte maturation, both BPA and BPS caused significant spindle abnormalities and chromosome misalignment, even at very low doses [78]. Both epidemiological and experimental evidence demonstrates that all bisphenol affects female infertility and/or subfertility.

\section{Phthalates}

The in vitro/vivo effects of phthalates on various reproductive organs have been extensively studied $[20,21,48,79]$. The relevance of these results to humans is still controversial. In women with infertility, urinary phthalates were found associated with decreased antral follicle count, which may lead to decreased fecundity [80]. In contrast, another study in women with a history of infertility did not show any difference in the concentrations of urinary phthalate metabolites [81]. A systematic review summarizing the evidence of the associations between common npEDCs and fecundability, and between phthalate exposure and time to pregnancy (TTP), only reported equivocal associations [82]. The studies on the effects of phthalates on fertility yielded heterogeneous results. These inconsistencies may be related to study designs and to the characteristics of the examined populations.

\section{Parabens and triclosan}

The Longitudinal Investigation of Fertility and the Environment (LIFE) study, which included 501 couples of reproductive age recruited in Michigan and Texas from 2005 and 2009, showed that exposure to high levels of methyl paraben and ethyl paraben was associated with reduced TTP [83]. According to LIFE study, exposure to parabens with longer TTP has been suggested. However, no effect of TCS on fecundability has been demonstrated [82].

\section{Conclusion}

The majority of the available data strengthen the evidence of reproductive health-related impact of $n p E D C s$ and we have summarized the published results about human on Table 1. Several animal studies and in vitro studies have shown that exposure to npEDCs can alter reproductive functions. However, the exact mechanisms by which npEDCs cause physiological, cellular, and molecular changes in women's reproductive health are not clear. Adverse effects can be caused by low-dose exposure and characterized by a non-linear dose response. Notably, these effects are not limited to the female reproductive age and can occur throughout the lifespan or through the generation. Recommendations should be made in order to reduce human exposure to npEDCs and to protect future generations from steadily increasing reproductive health risks.

\section{Acknowledgements}

This work was supported by the National Research Foundation of Korea (grant numbers: NRF-2018R1D1A1A02085827 and NRF-2016R1C1B1006976).

\section{Conflict of interest}

No potential conflict of interest relevant to this article was reported.

\section{References}

1. World Health Organization. State of the science of endocrine disrupting chemicals -2012. Geneva: World Health Organization; 2017.

2. Eskenazi B, Warner M, Brambilla P, Signorini S, Ames J, Mocarelli P. The Seveso accident: a look at 40 years of health research and beyond. Environ Int 2018;121:7184.

3. Saxena MC, Siddiqui MK, Seth TD, Krishna Murti CR, Bhargava AK, Kutty D. Organochlorine pesticides in specimens from women undergoing spontaneous abortion, premature of full-term delivery. J Anal Toxicol 1981;5:6-9.

4. Windham GC, Lee $D$, Mitchell $P$, Anderson $M$, Petreas $M$, Lasley B. Exposure to organochlorine compounds and 


\section{Obstetrics \& Gynecology Science}

Yeon Jean Cho, et al. npEDCs affect reproductive health

effects on ovarian function. Epidemiology 2005;16:18290.

5. Cooper GS, Savitz DA, Millikan R, Chiu Kit T. Organochlorine exposure and age at natural menopause. Epidemiology 2002;13:729-33.

6. Rogan WJ, Gladen BC, McKinney JD, Carreras N, Hardy $\mathrm{P}$, Thullen J, et al. Polychlorinated biphenyls (PCBs) and dichlorodiphenyl dichloroethene (DDE) in human milk: effects on growth, morbidity, and duration of lactation. Am J Public Health 1987;77:1294-7.

7. van Wendel de Joode $B$, Wesseling $C$, Kromhout $H$, Monge P, García M, Mergler D. Chronic nervous-system effects of long-term occupational exposure to DDT. Lancet 2001;357:1014-6.

8. Caserta D, Di Segni N, Mallozzi M, Giovanale V, Mantovani A, Marci R, et al. Bisphenol A and the female reproductive tract: an overview of recent laboratory evidence and epidemiological studies. Reprod Biol Endocrinol 2014;12:37.

9. Gore AC, Chappell VA, Fenton SE, Flaws JA, Nadal A, Prins GS, et al. EDC-2: The Endocrine Society's second scientific statement on endocrine-disrupting chemicals. Endocr Rev 2015;36:E1-150.

10. Wang W, Hafner KS, Flaws JA. In utero bisphenol A exposure disrupts germ cell nest breakdown and reduces fertility with age in the mouse. Toxicol Appl Pharmacol 2014;276:157-64.

11. Lee SG, Kim JY, Chung JY, Kim YJ, Park JE, Oh S, et al. Bisphenol A exposure during adulthood causes augmentation of follicular atresia and luteal regression by decreasing $17 \beta$-estradiol synthesis via downregulation of aromatase in rat ovary. Environ Health Perspect 2013;121:663-9.

12. Nah WH, Park MJ, Gye MC. Effects of early prepubertal exposure to bisphenol A on the onset of puberty, ovarian weights, and estrous cycle in female mice. Clin Exp Reprod Med 2011;38:75-81.

13. Monje L, Varayoud J, Muñoz-de-Toro M, Luque EH, Ramos JG. Exposure of neonatal female rats to bisphenol $A$ disrupts hypothalamic LHRH pre-mRNA processing and estrogen receptor alpha expression in nuclei controlling estrous cyclicity. Reprod Toxicol 2010;30:625-34.

14. Jukic AM, Calafat AM, McConnaughey DR, Longnecker MP, Hoppin JA, Weinberg CR, et al. Urinary concentrations of phthalate metabolites and bisphenol $A$ and associations with follicular-phase length, luteal-phase length, fecundability, and early pregnancy loss. Environ Health Perspect 2016;124:321-8.

15. Pollack AZ, Mumford SL, Krall JR, Carmichael AE, Sjaarda LA, Perkins NJ, et al. Exposure to bisphenol A, chlorophenols, benzophenones, and parabens in relation to reproductive hormones in healthy women: a chemical mixture approach. Environ Int 2018;120:137-44.

16. Svechnikova I, Svechnikov K, Söder O. The influence of di-(2-ethylhexyl) phthalate on steroidogenesis by the ovarian granulosa cells of immature female rats. J Endocrinol 2007;194:603-9.

17. Davis BJ, Maronpot RR, Heindel JJ. Di-(2-ethylhexyl) phthalate suppresses estradiol and ovulation in cycling rats. Toxicol Appl Pharmacol 1994;128:216-23.

18. Hannon PR, Peretz J, Flaws JA. Daily exposure to Di(2ethylhexyl) phthalate alters estrous cyclicity and accelerates primordial follicle recruitment potentially via dysregulation of the phosphatidylinositol 3-kinase signaling pathway in adult mice. Biol Reprod 2014;90:136.

19. Laskey JW, Berman E. Steroidogenic assessment using ovary culture in cycling rats: effects of bis(2-diethylhexyl) phthalate on ovarian steroid production. Reprod Toxicol 1993;7:25-33.

20. Gupta RK, Singh JM, Leslie TC, Meachum S, Flaws JA, Yao HH. Di-(2-ethylhexyl) phthalate and mono-(2ethylhexyl) phthalate inhibit growth and reduce estradiol levels of antral follicles in vitro. Toxicol Appl Pharmacol 2010;242:224-30.

21. Lovekamp-Swan T, Davis BJ. Mechanisms of phthalate ester toxicity in the female reproductive system. Environ Health Perspect 2003;111:139-45.

22. Baird DD, Saldana TM, Nepomnaschy PA, Hoppin JA, Longnecker MP, Weinberg $C R$, et al. Within-person variability in urinary phthalate metabolite concentrations: measurements from specimens after long-term frozen storage. J Expo Sci Environ Epidemiol 2010;20:169-75.

23. Nishihama $Y$, Yoshinaga J, lida A, Konishi S, Imai H, Yoneyama $\mathrm{M}$, et al. Association between paraben exposure and menstrual cycle in female university students in Japan. Reprod Toxicol 2016;63:107-13.

24. Vercellini P, Viganò P, Somigliana E, Fedele L. Endometriosis: pathogenesis and treatment. Nat Rev Endocrinol 2014; 10:261-75.

25. Crain DA, Janssen SJ, Edwards TM, Heindel J, Ho SM, 


\section{Obstetrics \& Gynecology Science}

Vol. 63, No. 1, 2020

Hunt $P$, et al. Female reproductive disorders: the roles of endocrine-disrupting compounds and developmental timing. Fertil Steril 2008;90:911-40.

26. Kendziorski JA, Belcher SM. Effects of whole life expo-

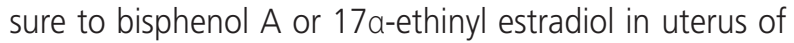
nulligravida CD1 mice. Data Brief 2015;5:948-53.

27. Kendziorski JA, Belcher SM. Strain-specific induction of endometrial periglandular fibrosis in mice exposed during adulthood to the endocrine disrupting chemical bisphenol A. Reprod Toxicol 2015;58:119-30.

28. Signorile PG, Spugnini EP, Mita L, Mellone P, D'Avino A, Bianco $M$, et al. Pre-natal exposure of mice to bisphenol A elicits an endometriosis-like phenotype in female offspring. Gen Comp Endocrinol 2010;168:318-25.

29. Signorile PG, Spugnini EP, Citro G, Viceconte R, Vincenzi $B$, Baldi $F$, et al. Endocrine disruptors in utero cause ovarian damages linked to endometriosis. Front Biosci (Elite Ed) 2012;4:1724-30.

30. Cobellis L, Colacurci N, Trabucco E, Carpentiero C, Grumetto $L$. Measurement of bisphenol $A$ and bisphenol $B$ levels in human blood sera from healthy and endometriotic women. Biomed Chromatogr 2009;23:1186-90.

31. Upson K, Sathyanarayana S, De Roos AJ, Koch HM, Scholes D, Holt VL. A population-based case-control study of urinary bisphenol A concentrations and risk of endometriosis. Hum Reprod 2014;29:2457-64.

32. Rashidi BH, Amanlou M, Lak TB, Ghazizadeh M, Eslami B. A case-control study of bisphenol $A$ and endometrioma among subgroup of Iranian women. J Res Med Sci 2017;22:7.

33. Simonelli A, Guadagni R, De Franciscis P, Colacurci $N$, Pieri M, Basilicata $P$, et al. Environmental and occupational exposure to bisphenol $A$ and endometriosis: urinary and peritoneal fluid concentration levels. Int Arch Occup Environ Health 2017;90:49-61.

34. Buck Louis GM, Peterson CM, Chen Z, Croughan M, Sundaram R, Stanford J, et al. Bisphenol A and phthalates and endometriosis: the endometriosis: natural history, diagnosis and outcomes study. Fertil Steril 2013;100:162-169.e1-2.

35. Itoh H, Iwasaki M, Hanaoka T, Sasaki H, Tanaka T, Tsugane $\mathrm{S}$. Urinary bisphenol-A concentration in infertile Japanese women and its association with endometriosis: a cross-sectional study. Environ Health Prev Med 2007;12:258-64.
36. Forte M, Mita L, Cobellis L, Merafina V, Specchio R, Rossi $S$, et al. Triclosan and bisphenol A affect decidualization of human endometrial stromal cells. Mol Cell Endocrinol 2016;422:74-83.

37. Cho YJ, Park SB, Park JW, Oh SR, Han M. Bisphenol A modulates inflammation and proliferation pathway in human endometrial stromal cells by inducing oxidative stress. Reprod Toxicol 2018;81:41-9.

38. Cobellis L, Latini G, De Felice C, Razzi S, Paris I, Ruggieri $F$, et al. High plasma concentrations of di-(2-ethylhexyl)phthalate in women with endometriosis. Hum Reprod 2003;18:1512-5.

39. Kim SH, Chun S, Jang JY, Chae HD, Kim CH, Kang BM. Increased plasma levels of phthalate esters in women with advanced-stage endometriosis: a prospective casecontrol study. Fertil Steril 2011;95:357-9.

40. Reddy BS, Rozati R, Reddy S, Kodampur S, Reddy P, Reddy R. High plasma concentrations of polychlorinated biphenyls and phthalate esters in women with endometriosis: a prospective case control study. Fertil Steril 2006;85:775-9.

41. Reddy BS, Rozati R, Reddy BV, Raman NV. Association of phthalate esters with endometriosis in Indian women. BJOG 2006;113:515-20.

42. Frederiksen H, Skakkebaek NE, Andersson AM. Metabolism of phthalates in humans. Mol Nutr Food Res 2007;51:899-911.

43. Weuve J, Hauser R, Calafat AM, Missmer SA, Wise LA. Association of exposure to phthalates with endometriosis and uterine leiomyomata: findings from NHANES, 1999-2004. Environ Health Perspect 2010;118:825-32.

44. Upson K, Sathyanarayana S, De Roos AJ, Thompson ML, Scholes $D$, Dills $R$, et al. Phthalates and risk of endometriosis. Environ Res 2013;126:91-7.

45. Itoh H, Iwasaki M, Hanaoka T, Sasaki H, Tanaka T, Tsugane $\mathrm{S}$. Urinary phthalate monoesters and endometriosis in infertile Japanese women. Sci Total Environ 2009;408:37-42.

46. Kim YH, Kim SH, Lee HW, Chae HD, Kim CH, Kang $\mathrm{BM}$. Increased viability of endometrial cells by in vitro treatment with di-(2-ethylhexyl) phthalate. Fertil Steril 2010;94:2413-6.

47. Kim SH, Cho S, Ihm HJ, Oh YS, Heo SH, Chun S, et al. Possible role of phthalate in the pathogenesis of endometriosis: in vitro, animal, and human data. J Clin Endo- 


\section{Obstetrics \& Gynecology Science}

Yeon Jean Cho, et al. npEDCs affect reproductive health

crinol Metab 2015;100:E1502-11.

48. Cho YJ, Park SB, Han M. Di-(2-ethylhexyl)-phthalate induces oxidative stress in human endometrial stromal cells in vitro. Mol Cell Endocrinol 2015;407:9-17.

49. Kim Y, Kim MR, Kim JH, Cho HH. Aldo-keto reductase activity after diethylhexyl phthalate exposure in eutopic and ectopic endometrial cells. Eur J Obstet Gynecol Reprod Biol 2017;215:215-9.

50. Flake GP, Andersen J, Dixon D. Etiology and pathogenesis of uterine leiomyomas: a review. Environ Health Perspect 2003;111:1037-54.

51. Shen $Y, X u$ Q, Ren $M$, Feng $X, C a i$, Gao Y. Measurement of phenolic environmental estrogens in women with uterine leiomyoma. PLoS One 2013;8:e79838.

52. Zhou F, Zhang L, Liu A, Shen Y, Yuan J, Yu X, et al. Measurement of phenolic environmental estrogens in human urine samples by HPLC-MS/MS and primary discussion the possible linkage with uterine leiomyoma. J Chromatogr B Analyt Technol Biomed Life Sci 2013;938:805.

53. Jeong EH, Hong GY, Kim BR, Park SN, Lee HH, Na YJ, et al. The relationship between uterine myoma growth and the endocrine disruptor in postmenopausal women. J Menopausal Med 2013;19:130-4.

54. Han MS, Byun JC, Park JE, Kim JY, Chung JY, Kim JM. Bisphenol-A concentrations from leiomyoma patients by LC/MS. Toxicol Res 2011;27:49-52.

55. Shen $Y$, Ren $M L$, Feng $X, C$ ai $Y L$, Gao $Y X, X u$ Q. An evidence in vitro for the influence of bisphenol $A$ on uterine leiomyoma. Eur J Obstet Gynecol Reprod Biol 2014;178:80-3.

56. Shen $Y$, Lu Q, Zhang P, Wu Y, Ren $M$. The effect of TGF- $\beta$ signaling on regulating proliferation of uterine leiomyoma cell via ERa signaling activated by bisphenol A, octylphenol and nonylphenol in vitro. J Cancer Res Ther 2018;14:S276-81.

57. Wang KH, Kao AP, Chang CC, Lin TC, Kuo TC. Bisphenol $A$ at environmentally relevant doses induces cyclooxygenase-2 expression and promotes invasion of human mesenchymal stem cells derived from uterine myoma tissue. Taiwan J Obstet Gynecol 2013;52:246-52.

58. Yu L, Das P, Vall AJ, Yan Y, Gao X, Sifre Ml, et al. Bisphenol $A$ induces human uterine leiomyoma cell proliferation through membrane-associated ERa36 via nongenomic signaling pathways. Mol Cell Endocrinol
2019;484:59-68.

59. Huang PC, Tsai EM, Li WF, Liao PC, Chung MC, Wang $\mathrm{YH}$, et al. Association between phthalate exposure and glutathione S-transferase M1 polymorphism in adenomyosis, leiomyoma and endometriosis. Hum Reprod 2010;25:986-94.

60. Kim YA, Kho Y, Chun KC, Koh JW, Park JW, BundersonSchelvan $\mathrm{M}$, et al. Increased urinary phthalate levels in women with uterine leiomyoma: a case-control study. Int J Environ Res Public Health 2016;13:E1247.

61. Zota AR, Geller RJ, Calafat AM, Marfori CQ, Baccarelli AA, Moawad GN. Phthalates exposure and uterine fibroid burden among women undergoing surgical treatment for fibroids: a preliminary study. Fertil Steril 2019;111:112-21.

62. Kim JH, Kim SH, Oh YS, Ihm HJ, Chae HD, Kim CH, et al. In vitro effects of phthalate esters in human myometrial and leiomyoma cells and increased urinary level of phthalate metabolite in women with uterine leiomyoma. Fertil Steril 2017;107:1061-1069.e1.

63. Takeuchi T, Tsutsumi O, Ikezuki Y, Takai Y, Taketani Y. Positive relationship between androgen and the endocrine disruptor, bisphenol $A$, in normal women and women with ovarian dysfunction. Endocr J 2004;51:1659.

64. Kandaraki E, Chatzigeorgiou A, Livadas S, Palioura E, Economou $F$, Koutsilieris $M$, et al. Endocrine disruptors and polycystic ovary syndrome (PCOS): elevated serum levels of bisphenol A in women with PCOS. J Clin Endocrinol Metab 2011;96:E480-4.

65. Tarantino G, Valentino R, Di Somma C, D'Esposito V, Passaretti F, Pizza $G$, et al. Bisphenol A in polycystic ovary syndrome and its association with liver-spleen axis. Clin Endocrinol (Oxf) 2013;78:447-53.

66. Vahedi M, Saeedi A, Poorbaghi SL, Sepehrimanesh M, Fattahi M. Metabolic and endocrine effects of bisphenol A exposure in market seller women with polycystic ovary syndrome. Environ Sci Pollut Res Int 2016;23:23546-50.

67. Hu Y, Wen S, Yuan D, Peng L, Zeng R, Yang Z, et al. The association between the environmental endocrine disruptor bisphenol A and polycystic ovary syndrome: a systematic review and meta-analysis. Gynecol Endocrinol 2018;34:370-7.

68. Rutkowska AZ, Diamanti-Kandarakis E. Polycystic ovary syndrome and environmental toxins. Fertil Steril 


\section{Obstetrics \& Gynecology Science}

Vol. 63, No. 1, 2020

2016;106:948-58.

69. Vagi SJ, Azziz-Baumgartner E, Sjodin A, Calafat AM, Dumesic D, Gonzalez $L$, et al. Exploring the potential association between brominated diphenyl ethers, polychlorinated biphenyls, organochlorine pesticides, perfluorinated compounds, phthalates, and bisphenol $A$ in polycystic ovary syndrome: a case-control study. BMC Endocr Disord 2014;14:86.

70. Hart R, Doherty DA, Frederiksen H, Keelan JA, Hickey $M$, Sloboda $D$, et al. The influence of antenatal exposure to phthalates on subsequent female reproductive development in adolescence: a pilot study. Reproduction 2014;147:379-90.

71. Hewlett M, Chow E, Aschengrau A, Mahalingaiah S. Prenatal exposure to endocrine disruptors: a developmental etiology for polycystic ovary syndrome. Reprod Sci 2017;24:19-27.

72. Ye J, Zhu W, Liu H, Mao Y, Jin F, Zhang J. Environmental exposure to triclosan and polycystic ovary syndrome: a cross-sectional study in China. BMJ Open 2018;8:e019707.

73. Gu J, Yuan T, Ni N, Ma Y, Shen Z, Yu X, et al. Urinary concentration of personal care products and polycystic ovary syndrome: a case-control study. Environ Res 2019;168:48-53.

74. Ziv-Gal A, Flaws JA. Evidence for bisphenol A-induced female infertility: a review (2007-2016). Fertil Steril 2016;106:827-56.

75. Huo X, Chen D, He Y, Zhu W, Zhou W, Zhang J. Bisphenol-A and female infertility: a possible role of geneenvironment interactions. Int J Environ Res Public Health 2015;12:11101-16.

76. Wang B, Zhou W, Zhu W, Chen L, Wang W, Tian Y, et al. Associations of female exposure to bisphenol A with fecundability: evidence from a preconception cohort study. Environ Int 2018;117:139-45.

77. Rochester JR, Bolden AL. Bisphenol S and F: a systematic review and comparison of the hormonal activity of bisphenol A substitutes. Environ Health Perspect 2015;123:643-50.

78. Campen KA, Kucharczyk KM, Bogin B, Ehrlich JM, Combelles CM. Spindle abnormalities and chromosome misalignment in bovine oocytes after exposure to low doses of bisphenol A or bisphenol S. Hum Reprod 2018;33:895-904.

79. Ahmad R, Verma Y, Gautam AK, Kumar S. Assessment of estrogenic potential of di-n-butyl phthalate and butyl benzyl phthalate in vivo. Toxicol Ind Health 2015;31:1296-303.

80. Messerlian C, Souter I, Gaskins AJ, Williams PL, Ford JB, $\mathrm{Chiu} \mathrm{YH}$, et al. Urinary phthalate metabolites and ovarian reserve among women seeking infertility care. Hum Reprod 2016;31:75-83.

81. Alur S, Wang H, Hoeger K, Swan SH, Sathyanarayana $\mathrm{S}$, Redmon BJ, et al. Urinary phthalate metabolite concentrations in relation to history of infertility and use of assisted reproductive technology. Fertil Steril 2015;104:1227-35.

82. Hipwell AE, Kahn LG, Factor-Litvak P, Porucznik CA, Siegel EL, Fichorova RN, et al. Exposure to non-persistent chemicals in consumer products and fecundability: a systematic review. Hum Reprod Update 2019;25:51-71.

83. Smarr MM, Sundaram R, Honda M, Kannan K, Louis GM. Urinary concentrations of parabens and other antimicrobial chemicals and their association with couples' fecundity. Environ Health Perspect 2017;125:730-6. 